\title{
Gratitud y felicidad en estudiantes del segundo al quinto año de una universidad privada de Lima este, 2016
}

\author{
Gratitude and happiness in students from the second to fifth year of a private university in Lima, \\ 2016
}

Susana Thalia Leon Lopez ${ }^{\star *}$, Ethel Priscilla Hidalgo Gómez², Maritza Mendoza Galarza

\begin{abstract}
RESUMEN
Objetivo: Determinar la relación entre las variables gratitud y felicidad en los estudiantes de una universidad privada de Lima este. El diseño de investigación es no experimental, de corte trasversal y de alcance correlacional. Materiales y Métodos: Los participantes fueron 404 estudiantes de ambos sexos entre las edades de 20 a 30 años. El instrumento empleado para la medición de la gratitud fue la Escala de gratitud, elaborada por Alarcón (2014) en Lima, Perú. Para medir la felicidad se utilizó la Escala de felicidad, elaborada por Alarcón (2006) en Lima, Perú, conformada por cuatro dimensiones: sentido positivo de la vida, satisfacción con la vida, realización personal y alegría de vivir. Resultados: Se relaciona significativamente con felicidad (rho=,324; $\mathrm{p}<0.01$ ), y con cada una de sus dimensiones. Conclusiones: Por lo tanto, se concluye que las personas que presentan un nivel elevado de gratitud tienen más probabilidad de experimentar la felicidad plena.
\end{abstract}

Palabras claves: Psicología positiva, gratitud, felicidad, sentido positivo de la vida, satisfacción con la vida, realización personal, alegría de vivir.

\begin{abstract}
Objective: To determine the relationship between the variables of gratitude and happiness in the students of a private university in East Lima. The research design is non-experimental, cross-sectional and correlational. Materials and Methods: The participants were 404 students of both sexes between the ages of 20 and 30 years old. The instrument used to measure gratitude was the Gratitude Scale, developed by Alarcón (2014) in Lima, Peru. In order to measure happiness, the Happiness Scale, elaborated by Alarcón (2006) in Lima, Peru, was used. It was integrated by four dimensions: positive meaning of life, satisfaction with life, personal fulfillment and joy of life. Results: It was significantly related to happiness (rho $=, 324 ; \mathrm{p}<0.01$ ), and with each of its dimensions. Conclusions: Therefore, it is concluded that people who have a high level of gratitude are more likely to experience full happiness.
\end{abstract}

Keywords: Positive psychology, gratitude, happiness, positive meaning of life, satisfaction with life, personal fulfillment, joy of life.

\footnotetext{
${ }^{1}$ Psicólogo, EP Psicología, Universidad Peruana Unión, Lima, Perú.

${ }^{2}$ Psicólogo, EP Psicología, Universidad Peruana Unión, Lima, Perú.

${ }^{3}$ Docente de Psicología, Facultad de Ciencias de la Salud, Universidad Peruana Unión, Lima, Perú.
} 


\section{INTRODUCCIÓN}

La búsqueda de la felicidad ha constituido una de las principales preocupaciones del ser humano, y aún hoy, esta búsqueda sigue siendo relevante, pues forma parte de su esencia.

La humanidad está inmersa en el progreso tecnológico, vive de manera acelerada una modernidad definida por la explosión del consumo y la defensa del individualismo.

Todo lo mencionado hace que la satisfacción de los placeres sensoriales se asemeje a la auténtica felicidad. Sin embargo, aquel placer consumista no proporciona felicidad (Román y Montero, 2013).

Frente a esa necesidad, Helliwell, Layard y Sachs (2015) realizaron y publicaron la cuarta edición del reporte mundial de felicidad, en el que se mide el bienestar conforme a una serie de variables estadísticas y percepciones individuales como son: la percepción de la corrupción, el apoyo social, la libertad, la generosidad, la expectativa de vida y el producto de interior bruto (PIB) per-cápita. Los resultados encontrados muestran a Europa y Latinoamérica como los continentes más felices, siendo Suiza el país que se encuentra en la cima, seguida de cerca por Islandia, Dinamarca y Noruega. Por otro lado, en Latinoamérica, Costa Rica es considerado el país más feliz, situado en el puesto 12, seguida de México, Brasil, Panamá, Chile y Argentina; sin embargo, Perú se encontró en el lugar 58, entre los cinco países menos felices de América Latina (Helliwell et al., 2015).

Por lo descrito anteriormente, la felicidad es y ha sido una necesidad difícil de cubrir. En este sentido, arragona (2012) encontró que existen variables que se correlacionan fuertemente con la felicidad como son: la resiliencia, el optimismo, el trabajo, área sexual, afectos positivos percibidos, autoestima y la gratitud. Esta última variable es un factor importante para obtener el bienestar y felicidad plena (Watkins, 2004).

Sin embargo, los estudios orientados al análisis de la relación entre gratitud y felicidad son escasos. Existe por ejemplo el estudio realizado por Alarcón y Caycho (2015), donde se evidencian que estas dos variables correlacionan positiva y significativamente, lo que quiere decir que las personas agradecidas tienden a experimentar una mayor felicidad. En otros estudios, Emmons y Sheldon (2005) indican que existe relación positiva entre gratitud y el bienestar subjetivo, donde realizaron un ejercicio para analizar los efectos de llevar un diario de gratitud durante 21 días, encontrando que las personas mostraban mejor salud emocional y sentían más apoyo y ayuda de los demás. Así también, Emmons y McCullough (2003) señalaron que las personas gratas son más propensas a experimentar mayores emociones positivas y una mayor felicidad.

La investigación tiene como objetivo determinar la relación entre gratitud y felicidad en estudiantes de una universidad privada de Lima este. Por lo cual, se pretende explicar los objetivos generales respondiendo las siguientes preguntas: ¿existe relación significativa entre gratitud y sentido positivo de la vida?, ¿gratitud y satisfacción con la vida se relacionan significativamente?, ¿hay relación entre gratitud y realización personal?, ¿gratitud y alegría de vivir son factores que se relacionan entre sí?

\section{MATERIALES Y MÉTODOS}

Para llevar a cabo la investigación se utilizó el diseño no experimental de corte transversal y de tipo correlacional, porque el objetivo de la investigación es relacionar las variables de estudio.

Los participantes fueron 404 estudiantes de una universidad privada de Lima este, con edades oscilantes entre 20 y 30 años, que se encontraban cursando entre el segundo y quinto año, seleccionados a través de un muestreo no probabilístico intencional, quienes completaron las escalas de forma voluntaria.

Los componentes del cuestionario incluyeron una declaración de consentimiento informado, un cuestionario de elaboración propia (datos sociodemográficos), las instrucciones, Escala de Gratitud de Lima y Escala de Felicidad de Lima.

\section{Cuestionario de datos sociodemográficos}

Recoge información referente a edad, género, estado civil, año académico que cursaba, facultad a la que pertenece, religión, nivel socioeconómico y lugar de procedencia.

\section{Escala de Gratitud de Lima}

Fue elaborado por Alarcón (2014), constituida por tres dimensiones: reciprocidad, obligación moral y cualidad sentimental. Contiene 18 ítems con una escala de respuestas de tipo Likert que van desde Totalmente en desacuerdo hasta Totalmente de acuerdo, con una puntuación de 1 a 5 puntos respectivamente, en donde al extremo negativo se le otorga 1 punto y al extremo positivo 5 puntos. Los ítems 05, 06 y 11 tienen 
calificación inversa. Para esta investigación se utilizó el Alfa de Cronbach, con un resultado $\alpha=, 851$, la cual es indicador de una elevada fiabilidad. Asimismo, la validez de constructo se obtuvo mediante el método de análisis de subtest - test, donde los coeficientes de correlación son significativas.

\section{Escala de Felicidad de Lima}

Fue elaborado por Alarcón (2006), constituida por cuatro dimensiones: sentido positivo de la vida, satisfacción con la vida, realización personal y alegría de vivir. Contiene 27 ítems con una escala de respuestas de tipo Likert que va desde Totalmente en desacuerdo hasta Totalmente de acuerdo, en donde al extremo negativo se le otorga 1 punto y al extremo positivo 5 puntos. Los ítems 02, 07, 14, 17, 18, 19, 20, 22, 23 y 26 tienen calificación inversa. Para esta investigación se utilizó el Alfa de Cronbach, con un resultado $\alpha=, 920$, lo que indica elevada fiabilidad. Además, la validez de constructo se obtuvo mediante el método de análisis de subtest - test, donde los coeficientes de correlación son significativas.

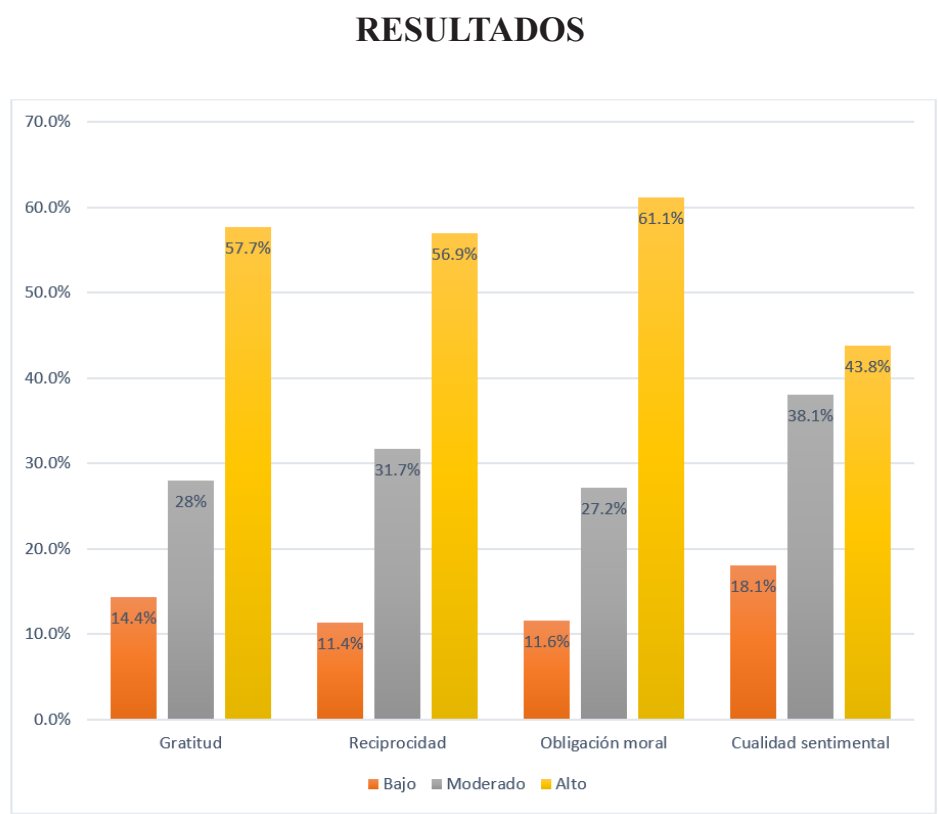

Figura 1. Niveles de gratitud en estudiantes universitarios de 20 a 30 años.

En la Figura 1 se observa que el 57.7\% de la población total, se encuentra en el nivel alto de gratitud. Del mismo modo, se evidencia que en la dimensión de obligación moral los participantes obtienen un nivel alto $(61.1 \%)$.

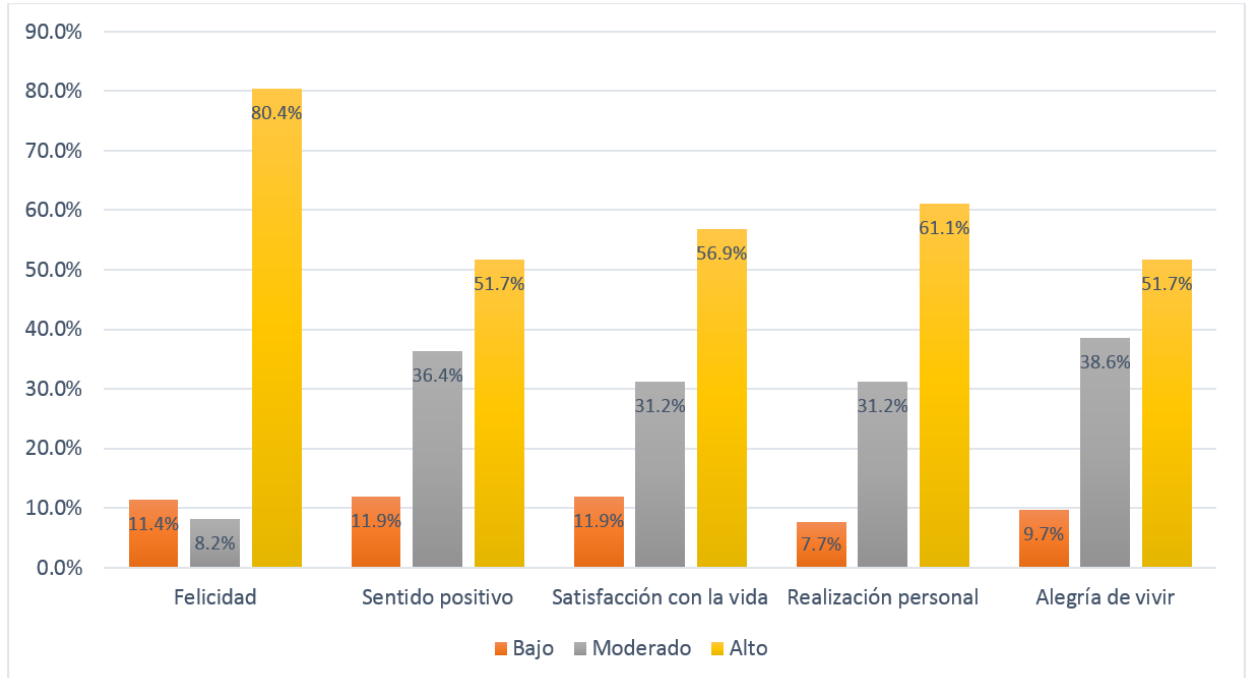

Figura 2. Niveles de felicidad en estudiantes universitarios de 20 a 30 años. 
La figura 2 muestra que, de la población total, el $80.4 \%$ se encuentra en el nivel alto de felicidad. Asimismo, se aprecia que en la dimensión de realización personal los participantes obtienen un nivel alto $(61.1 \%)$.

Tabla 1

Coeficiente de correlación entre felicidad y gratitud

\begin{tabular}{lcc}
\hline \multirow{2}{*}{ Felicidad } & \multicolumn{2}{c}{ Gratitud } \\
\cline { 2 - 3 } & rho & p \\
\hline Felicidad &, $324^{* *}$ &, 000 \\
Sentido positivo de la vida &, $445^{* *}$ &, 000 \\
Satisfacción con la vida &, $355^{* *}$ &, 000 \\
Realización personal &, $184^{* *}$ &, 000 \\
Alegría de vivir &, $368^{* *}$ & \\
\hline
\end{tabular}

** La correlación es significativa al nivel 0.01

Como se puede apreciar en la Tabla 2, el coeficiente de correlación de Spearman indica que existe una relación moderada positiva y altamente significativa entre el nivel de felicidad total y gratitud $(\mathrm{rho}=, 324$; $\mathrm{p}<0.01$ ). Asimismo, se aprecia que la dimensión de sentido positivo de la vida presenta una relación moderada positiva y altamente significativa con relación a la gratitud $(\mathrm{r}=, 445 ; \mathrm{p}<0.01)$.

\section{DISCUSIÓN}

En cuanto al análisis de relación entre gratitud y felicidad, en el estudio se corroboró que si existe relación altamente significativa y positiva entre las variables de estudio ( $\mathrm{rho}=, 324 ; \mathrm{p}<0.01$ ), es decir que a mayor gratitud mayor grado de felicidad experimentan los estudiantes universitarios. Similares resultados fueron encontrados en el estudio realizado por Alarcón y Caycho (2015) en el Perú, donde la gratitud y felicidad correlacionan positiva y significativamente. Entendiendo que las personas agradecidas tienden a desarrollar un mayor grado de emociones positivas, presentando características pro sociales como empatía, capacidad para perdonar, servicio y demuestran apoyo emocional hacia los demás, es así que la gratitud motiva el comportamiento prosocial, por lo que las personas con estas características están más relacionadas con el afecto positivo, el bienestar y la felicidad. Asimismo, los jóvenes que tienen el agrado y la satisfacción de corresponder a una persona de quien ha recibido un favor, tienen más probabilidad de experimentar alegría, satisfacción consigo mismo y perciben la vida como maravillosa (McCullough, Emmons y Tsang, 2002; McCullough et al., 2001; Alarcón y Caycho, 2015; Diener, 2000).
Además, se encontró relación altamente significativa entre gratitud y sentido positivo de la vida $(\mathrm{rho}=, 445$; $\mathrm{p}<0.01$ ). Esto coincide con el estudio realizado por Lambert, Fincham y Stillman (2012) quienes demostraron que existe relación entre ellas, señalando que la gratitud impulsa a las personas a experimentar un sentido positivo de la vida, a través del cambio de las experiencias negativas por experiencias potencialmente positivas, tales como felicidad, libertad y cumplimiento de metas vitales, que a su vez manifestaran emociones positivas (alegría, amor, humor, fluidez elevación y creatividad) lo que disminuyen los síntomas depresivos (vacío existencial, pesimismo, tristeza, desesperanza). Según el enfoque positivista, Seligman (2002) refiere que el sentido positivo de la vida se genera a través de las emociones positivas que experimenta el ser humano; en base a ello, Fredrickson (2009) postuló que la gratitud es una de las diez emociones positivas, frecuente en la vida cotidiana de las personas y que permite tener un sentido positivo a la vida. Es decir, los estudiantes que mostraron alegría, afecto, humor, fluidez y creatividad como emociones positivas, también demostraron tener una conducta agradecida, reflejando actitudes, experiencias y sentimientos positivos hacia sí mismo y hacia la vida, lo que significa estar libre de estados depresivos profundos, como menciona Alarcón (2006) el sentido positivo de la vida es la ausencia de sufrimiento.

Del mismo modo, gratitud y satisfacción con la vida están relacionadas significativamente $(\mathrm{rho}=, 355$; $\mathrm{p}<0.01)$. En una investigación realizada por Froh, Emmons, Card, Bono y Wilson (2011)035 se encontró que la gratitud se relacionó con diversos factores, entre ellos la satisfacción con la vida, evidenciándose que los jóvenes en estudio eran más agradecidos y 
a su vez, estaban más satisfechos con la vida (Froh, Sefick y Emmons, 2008; Froh, Yurkewicz y Kashdan, 2009; Emmons, 2007; Alarcón y Caycho, 2015). Para Csikszentmihalyi (2004) un factor que contribuye a la satisfacción con la vida es realizar actividades que representen retos en las que sea necesario una concentración completa, y que requiera el desarrollo de habilidades, experimentando gozo (flujo) más que experiencias placenteras (hedonismo).

En relación a ello, Seligman (2003) señaló que al experimentar gozo, se aprecia los aspectos buenos de la vida y se percibe satisfacción con la misma, que son respuestas emocionales que acompañan el reconocimiento típico de agradecimiento, que a su vez permite el desarrollo de gratitud por los logros alcanzados. Es decir que la satisfacción con la vida es considerada un aspecto afectivo de la gratitud.

Así también, en cuanto a la relación entre la dimensión de realización personal y gratitud, el coeficiente rho de Spearman muestra un grado de intensidad de $(\mathrm{rho}=.184$, $\mathrm{p}=0.000$ ), que indica que la relación es débil pero altamente significativa. En cuanto a la relación Maslow (1973) menciona que una persona que haya alcanzado la realización personal presenta 16 características y una de ellas es la necesidad de transcendencia, que hace referencia a la necesidad de contribuir a la humanidad por medio del servicio y la gratitud y tener un sentido de obligación moral hacia otros.

Esto coincide con el aporte de Peterson y Seligman (2004) desde el enfoque de la psicología positiva, en el cual mencionan que si una persona evidencia virtud de trascendencia, es decir que se conecte con el universo y le dé significado a su vida, experimentará gratitud; lo que explica que una persona que culmine el proceso de autorrealización, tiende a desarrollar características que harán de él una persona grata. Sin embargo, en la presente investigación los resultados evidencian relación débil, esto podría ser explicado por Vaillant (1979) y Huberman (1974) quienes manifiestan que los jóvenes de 20 a 30 años están en la etapa de establecimiento que se caracterizan por estar enfocados en uno mismo y satisfacer sus necesidades personales, tales como seguir su identidad social a través del trabajo, carrera y oficio, lograr un puesto determinado, conseguir una ideología propia, elegir al compañero para su vida y presentar una buena imagen de sí mismo. Según Maslow (1973) estas características pertenecen al primer proceso para alcanzar la autorrealización, por lo tanto la gratitud podría experimentarse en niveles bajos.

Igualmente, gratitud y alegría de vivir se encuentran relacionadas significativamente $(, 368 ; \mathrm{p}<0.01)$.
Como lo menciona Froh, Yurkewicz y Kashdan (2009) en su estudio, el estado de ánimo agradecido indica mayor bienestar subjetivo, en el que se experimenta niveles altos de felicidad y optimismo; es decir, los estudiantes al ser gratos con los demás, son conscientes de todo lo bueno que les suceden y a su vez, sienten que la vida es maravillosa, rescatando experiencias positivas de ella; lo que conlleva a manifestar agradecimiento, apreciación a la vida, sensación de bienestar, y por ende, tener una vida alegre y llena de optimismo, donde se espera que el futuro ofrezca resultados favorables y logren resolver los problemas con actitud positiva (McCullough et al., 2001; Watkins, 2004; Alarcón y Caycho, 2015).

Alarcón (2006) desarrolló la dimensión de alegría de vivir en base a los indicadores que señalan lo maravilloso que es vivir, sentirse generalmente bien y manifestar alegría. Por su parte, Seligman (2002) refirió que la alegría construye en el individuo diferentes habilidades, tales como: la creatividad, amplía la flexibilidad de pensamiento y el lado artístico, las cuales se convierten en recursos perdurables y pueden utilizarse a lo largo de la vida. Según Fredrickson (2009), la alegría y la gratitud pertenecen a las diez emociones positivas (gozo, serenidad, interés, esperanza, orgullo, diversión, inspiración, asombro y amor), que son frecuentes y necesarias en el ser humano, en este sentido se señala que el desarrollo continuo de alegría y gratitud, permitirá al ser humano vivir experiencias positivas, favoreciendo el bienestar emocional, desarrollo de la resiliencia y ampliación de pensamiento.

\section{Declaración de financiamiento y de conflicto de intereses:}

El estudio fue financiado por los autores, quienes declaran no tener algún tipo de conflicto de interés en la investigación realizada.

\section{Correspondencia}

Maritza Mendoza Galarza

Universidad Peruana Unión

Carretera Central Km 19.5 Ñaña, Lima, Perú

e-mail: maritzaj@upeu.edu.pe 


\section{REFERENCIAS BIBLIOGRÁFICAS}

Ardila, R. (2003). Calidad de vida: Una definición integradora. Revista Latinoamericana de Psicologia, 35(2), 161-164.

Brito, J., Jiménez, K., Tolorza, G., Siqués, P., Rojas, F., \& Barrios, L. (2007). Impacto de la ostomía en el paciente y su entorno. Revista Chilena de Cirugía, 56(1), 31-34.

Castro, M., Castro, C., \& Clemente, H. (2013). Los cuidados de enfermería en el impacto psicológico del paciente. Ene. Revista de Enfermeria, 7(3).

Casado, C. (2012). Plan de cuidados. Cáncer de recto: paciente colostomizado. Rev. Reduca (Enfermería, Fisioterapia y Podología), 4(1) 563-596. Recuperado de http://www. revistareduca.es/index.php/reduca-enfermeria/article/ viewFile/1009/1021

Castañeda, O. C., Garduño, M. A. C., Castillo, L. P., Bautista, M. D. P. S., Estévez, J. M. E., Espinosa, J. G. R., \& Corral, S. H. (2009). Epidemiología de ostomías de eliminación en diferentes unidades de salud del Distrito Federal. Revista CONAMED, 14(1), 15-19.

Cesaretti, I. U. R., Santos, V. L. C. G., \& Vianna, L. A. C. (2010). Qualidade de vida de pessoas colostomizadas com e sem uso de métodos de controle intestinal. Revista Brasileira de Enfermagem, 63(1), 16-21. http:// doi.org/10.1590/S0034-71672010000100003.

Crespo, L. (2012). Plan de cuidados de enfermería estandarizados en el paciente ciolostomizado. Recuperado de http://repositorio.unican.es/xmlui/ bitstream/handle/10902/3940/CrespoVillazanL. pdf? sequence $=1$.

Carrasco, S. (2005). Metodología de la investigación científica, pautas metodológicas para diseñar y elaborar el proyecto de investigación. Lima-Perú: San Marcos.

Charúa-Guindic, L., Benavides-león, C. J., Villanuevaherrero, J. A., Jiménez-bobadilla, B., Abdo-francis, J. M., \& Hernández-labra, E. (2011). Calidad de vida del paciente ostomizado. Cirugía Digestiva, 79, 149-155.

Costa, A., \& Parrelli, M. (2011). La sexualidad del paciente con ostomía: revisión integradora. Revista de Enfermagem, 7, 4935-4943. http://doi.org/10.5205/ reuol.4700-39563-1-ED.0707esp201315.

Ferreira-Umpiérrez,A.H. (2013). Viviendo con una ostomía: Percepciones y expectativas desde la fenomenología social. Texto E Contexto Enfermagem, 22(3), 687-694. http://doi.org/10.1590/S0104-07072013000300015.

Gonzalo, m. (2009). percepcion de la calidad de vida en el paciente colostomizado permanentemente. Journal of Coloproctology, 3(2), 70-75.

Hernández, F. (2011). Impacto de un plan de intervención educativa en la calidad de vida del paciente ostomizado. Rev. Reduca, 3(2), 504-533. Recuperado de http:// www.revistareduca.es/index.php/reduca-enfermeria/ article/viewFile/764/780.
Hernández, R., Fernández, C., \& Baptista, M. (2010). Metodología de la investigación. México: McGrawHill Interamericana.

Espinoza Herrera, M. (2003). Conocimientos y prácticas sobre el autocuidado que tienen los pacientes colostomizados que asisten a consulta de enfermería del hospital nacional Edgardo Rebagliati Martins. (Tesis para optar la licenciatura en enfermería). Recuperado de http://sisbib.unmsm.edu.pe/bibvirtualdata/tesis/salud/ espinoza_hm/enpdf/t_completo.pdf

Kimura, C. A., Kamada, I., Guilhem, D., \& Fortes, R. C. (2013). Perception of sexual activities and the care process in ostomized women. Journal of Coloproctology, 33(3), 145-150. http://doi. org/10.1016/j.jcol.2013.05.004.

OMS (2015). Organización Mundial de la Salud. Estrategia mundia sobre la percepcion de la calidad de vida.

Pita, P. et al. (2015). Caso clínico de dermatitis periestomal y dehiscencia en una colostomía. Rev. Enferm Dermatol, 9(24). Recuperado de http://dialnet.unirioja.es/servlet/ articulo? codigo $=5185639$

Sánchez, H. \& Reyes, C. (2002). Metodología y diseño en la investigación científica. Lima-Perú: Editorial Universitaria. Universidad Ricardo Palma.

Villajuan, E. (2006). calidad de vida de pacientes Ostomizado que asisten a consulta de Enfermería del Instituto Nacional de Enfermedades Neoplásicas. Revista Facultad Nacional de Salud Publica, 61(3). http://doi.org/10.4067/S0718-40262009000300008

Xiuxiu, Q. (2012). Quality of life in rectal cancer patients with permanent colostomy in Xi'an. African Health Sciences, 14(1) 28-36. Recuperado de

Recibido: 26/02/2017 Aceptado: 20/04/2017 